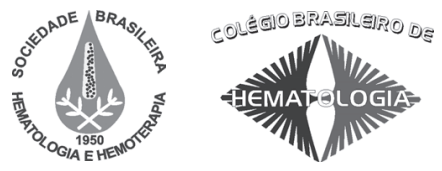

\title{
Linfoma de Hodgkin: aspectos atuais
}

\section{Hodgkin lymphoma: current issues}

Nelson Spector

\begin{abstract}
A par dos extraordinários avanços obtidos no tratamento do linfoma de Hodgkin, diversos desafios persistem na compreensão da biologia da doença, e na determinação de alternativas que maximizem a eficácia terapêutica e minimizem as toxicidades imediatas e tardias. O objetivo deste artigo é apresentar informações recentes que têm relevância imediata para aqueles que cuidam de pacientes com linfoma de Hodgkin (LH). Rev. Bras. Hematol. Hemoter. 2009;31(Supl. 2):3-6.
\end{abstract}

Key words: Linfoma de Hodgkin; tratamento; idosos; gravidez.

\section{Introdução}

A par dos extraordinários avanços obtidos no tratamento do linfoma de Hodgkin, diversos desafios persistem na compreensão da biologia da doença e na determinação de alternativas que maximizem a eficácia terapêutica e minimizem as toxicidades imediatas e tardias.

O objetivo deste artigo é apresentar informações recentes que têm relevância imediata para aqueles que cuidam de pacientes com linfoma de Hodgkin (LH).

\section{O linfoma de Hodgkin com predomínio infocítico nodular}

O linfoma de Hodgkin com predomínio linfocítico nodular responde por aproximadamente $5 \%$ de todos os LH. $\mathrm{O}$ diagnóstico exige confirmação por patologista experimentado, pois no maior estudo já realizado, com 426 pacientes inicialmente incluídos em 17 países da América do Norte e Europa, $49 \%$ dos casos foram reclassificados após a revisão histopatológica. ${ }^{1}$

A doença é localizada e assintomática ao diagnóstico na maioria dos casos. O curso clínico se caracteriza por altas taxas de resposta, tendência a recaídas tardias e risco de progressão histológica para linfoma de grandes células. Estudos recentes evidenciam que a probabilidade de progressão histológica atinge $12 \%$ em dez anos. ${ }^{2}$ A sobrevida, após a transformação, é semelhante à do linfoma difuso de grandes células.
Por este motivo, a abordagem mais conservadora da doença localizada (watch and wait) não é recomendada. Em crianças e adolescentes, muitos propõem o tratamento local, se possível com excisão cirúrgica completa, seguida de monitoramento clínico. Em adultos, pacientes com doença em estádio IA podem ser tratados com radioterapia dos campos envolvidos, seguida de monitoramento clínico. Outra opção que apresentou bons resultados nestes casos, embora mais dispendiosa, é o rituximabe. Pacientes com doença em outros estádios devem ser tratados de forma semelhante às formas clássicas do LH.

\section{Papel e limitações do PET no linfoma de Hodgkin}

O LH apresenta boa avidez pelo marcador metabólico radiativo (F18-deoxiglicose) usado na tomografia por emissão de pósitrons (positron emission tomography, PET). No entanto, é essencial ter em mente que resultados negativos com o PET são mais confiáveis do que resultados positivos. $\mathrm{O}$ valor preditivo de um exame negativo varia entre $80 \% \mathrm{e}$ $100 \%$ em diversas séries, ao passo que o valor preditivo de um exame positivo varia muito mais, de $45 \%$ a $100 \% .{ }^{3}$ Em outras palavras, em algumas séries, até metade dos pacientes com captação ao PET não apresentam efetivamente doença ativa, quando a área captante é biopsiada e avaliada ao microscópio.

A primeira indicação para a qual o PET comprovou sua utilidade foi a avaliação dos casos em que persiste imagem

Prof. Titular de Hematologia, Faculdade de Medicina, UFRJ

Faculdade de Medicina, Universidade Federal do Rio de Janeiro - Rio de Janeiro-RJ.

Correspondência: Nelson Spector

E-mail:nelson.spector@gmail.com 
residual ao final do tratamento. Caso a lesão residual não capte o marcador, a probabilidade de doença ativa é muito baixa, e o paciente pode ser apenas observado.

Mais recentemente, diversos estudos verificaram que o PET tem grande potencial na identificação precoce do prognóstico de pacientes durante o tratamento do LH. Em estudo que reuniu a experiência dos grupos cooperativos da Itália e Dinamarca, a sobrevida livre de progressão em dois anos dos pacientes com PET positivo após dois ciclos de tratamento foi de $13 \%$, contra $95 \%$ nos pacientes com PET negativo. ${ }^{4}$

O Gruppo Italiano Terapie Innovative nei Linfomi apresentou recentemente os resultados da intensificação do tratamento com BEACOPP escalado em pacientes com LH avançado e PET positivo após dois ciclos de ABVD. ${ }^{5}$ Entre os 24 pacientes com PET positivo, a sobrevida livre de progressão após o tratamento com BEACOPP foi de $56 \%$ em dois anos, resultado substancialmente superior à experiência anterior $(13 \%) .{ }^{5}$ Se confirmada, esta abordagem traria enormes vantagens, pois permitiria restringir o tratamento BEACOPP aos pacientes que efetivamente necessitem.

Diversos estudos em andamento incluem um braço controle, o que permitirá definir melhor, nos próximos anos, o impacto dessa estratégia que por ora permanece restrita aos estudos clínicos.

Outra dificuldade no PET é a baixa taxa de concordância entre os experts. Diversas iniciativas estão em curso para padronizar os critérios de avaliação do PET em linfomas, e para criar mecanismos de revisão centralizada em tempo real dos exames no âmbito dos estudos clínicos. ${ }^{6}$

\section{Tratamento da doença localizada}

A abordagem do paciente com doença localizada vem sendo refinada por meio de importantes estudos clínicos cooperativos. Consolidou-se entre os grupos europeus o conceito de que o LH localizado deve ser dividido em casos de risco favorável e desfavorável, de acordo com critérios clínicos bem estabelecidos. ${ }^{7}$

A administração de quimioterapia seguida de radioterapia, o chamado tratamento combinado, tornou-se a alternativa mais empregada na doença localizada. Em pacientes com doença de risco favorável, um importante estudo do German Hodgkin Study Group, o HD10, foi desenvolvido para comparar dois ou quatro ciclos de $\mathrm{ABVD}$, seguidos de radioterapia com 20 Gy ou 30 Gy sobre os campos inicialmente envolvidos. O estudo já recrutou os 1.375 pacientes planejados, que vêm sendo acompanhados. Dados divulgados em análises interinas sugerem que não há diferenças em nenhum desfecho terapêutico entre os grupos, o que favorece a administração de apenas dois ciclos de ABVD seguidos de tãosomente 20 Gy nos campos envolvidos. ${ }^{8}$

$\mathrm{Na}$ doença localizada de alto risco, o estudo HD14 do grupo alemão foi selecionado entre os cinco melhores traba- 1hos do congresso europeu de hematologia de 2009. ${ }^{9}$ Esse estudo comparou a administração de quatro ciclos de ABVD com a administração de dois ciclos de BEACOPP escalado seguida de dois ciclos de ABVD. Em ambos os grupos, o tratamento foi consolidado com radioterapia 30 Gy sobre os campos envolvidos. A sobrevida livre de progressão em três anos foi de $90 \%$ versus $96 \%$. No entanto, a taxa de infertilidade resultante deste tratamento não foi ainda divulgada. Sabe-se que, após seis ciclos, o regime BEACOPP escalado produz infertilidade na maioria dos pacientes de ambos os sexos.

Ao mesmo tempo, diversos estudos demonstraram nos últimos anos a possibilidade de tratar a doença localizada tão-somente com quimioterapia, com resultados equivalentes aos do tratamento combinado. ${ }^{10,11}$ Esta abordagem pode trazer vantagens, especialmente nos casos em que seria necessário irradiar o tórax ou regiões cervicais altas e submandibulares, com riscos de sérias complicações tardias.

Finalmente, uma abordagem radioterápica ainda mais econômica vem sendo avaliada nos últimos anos: a radioterapia restrita aos gânglios linfáticos envolvidos (involvednodal radiation therapy). $\mathrm{O}$ tratamento é direcionado para o volume ganglionar presente antes da quimioterapia, e leva em conta a margem de deslocamento dos gânglios linfáticos. A abordagem exige um mapeamento meticuloso de todos os gânglios envolvidos, com PET, antes do início do tratamento, mas parece proporcionar resultados equivalentes, com menor exposição dos tecidos normais à irradiação. ${ }^{12}$

\section{É possível aperfeiçoar o ABVD?}

O ABVD é ainda considerado o padrão de tratamento do LH contra o qual os outros tratamentos devem ser comparados. Recentemente, o German Hodgkin Study Group divulgou os resultados preliminares (safety analysis) de um grande estudo em pacientes com doença localizada (HD13), no qual os pacientes são randomizados para tratamento em quatro grupos: $\mathrm{ABVD}, \mathrm{AVD}, \mathrm{ABV}$ ou $\mathrm{AV}$. Os pacientes tratados com $\mathrm{ABV}$ e com $\mathrm{AV}$ apresentaram resultados iniciais inferiores, que levaram à suspensão desses dois braços do estudo. ${ }^{7}$ Caso o AVD venha a apresentar resultados equivalentes ao ABVD, os pacientes poderiam ser poupados da potencial toxicidade pulmonar da bleomicina, que atinge, em algumas séries, $27 \%$ dos pacientes tratados, com $5 \%$ de eventos fatais. ${ }^{13}$

Evidências recentes sugerem que o ABVD pode ser integralmente administrado na dose calculada, sem necessidade de correção de doses em função de citopenias, e sem necessidade de G-CSF. ${ }^{14}$ Muitos clínicos experientes vêm adotando esta conduta, que proporciona intensidade de dose máxima. A taxa de toxicidade pulmonar na experiência publicada foi baixa, o que corrobora evidências anteriores de que esta toxicidade da bleomicina é potencializada pela administração de G-CSF. 


\section{Tratamento de idosos com linfoma de Hodgkin}

Os resultados do tratamento do $\mathrm{LH}$ em indivíduos com idade superior a 60 anos são piores em todo o mundo. A sobrevida global em cinco anos, que é superior a $80 \%$ nos pacientes com menos de 60 anos, não atinge sequer 50\% nos mais idosos. ${ }^{15}$ As causas dessa diferença são diversas e não estão inteiramente estabelecidas. A presença de comorbidades pode levar à redução da intensidade terapêutica, por vezes apropriada, outras vezes desnecessária. É importante ressaltar que o regime BEACOPP está formalmente contra-indicado nessa faixa etária, devido à alta taxa de complicações imediatas. Alguns centros utilizam o etoposide no lugar da doxorrubicina em pacientes com disfunção cardíaca, mas não há comprovação de sua equivalência. Da mesma forma, outros têm administrado o AVD, sem bleomicina, em pacientes com disfunção respiratória. Há um interesse renovado no tratamento do idoso com LH, e diversos estudos clínicos estão em andamento.

\section{Tratamento da grávida com linfoma de Hodgkin}

Recentemente, o BCCC apresentou a sua experiência no manejo de grávidas que desenvolvem LH. ${ }^{16}$ Entre os aspectos peculiares do manejo dessas pacientes, destacamse a recomendação de evitar radiografias ou tomografias computadorizadas, com a exceção de uma radiografia simples de tórax em PA, com blindagem adequada do abdomen. Ultrassonografia abdominal para avaliação retroperitoneal é recomendada.

Se for possível, a paciente deve ser acompanhada, sem tratamento do LH, até o termo. Caso haja evidências de atividade da doença, a administração intermitente de vinblastina permite o controle clínico até o parto. Nas pacientes tratadas com vinblastina o estadiamento fica prejudicado, e elas devem ser tratadas após o parto com seis a oito ciclos de quimioterapia. As outras pacientes devem completar o estadiamento após o parto.

Em 17 casos assim tratados em Vancouver, 11 permaneceram sem tratamento até o parto, e seis foram tratadas com vinblastina. Todas as 17 crianças nasceram e cresceram sem problemas de saúde. Entre as mães, 13 estão vivas em remissão completa, e quatro morreram.

Estes são, em uma exposição sumária, alguns dos aspectos mais atuais da abordagem clínica do LH. Diversos estudos em andamento deverão proporcionar, nos próximos anos, um ajuste fino do tratamento desta doença, que representa uma das histórias de maior sucesso da medicina moderna.

\section{Abstract}

Knowing the extraordinary advances obtained in the treatment of Hodgkin's lymphoma, several challenges persist related to the biology of the disease and to the determination of alternatives that maximize the therapeutic efficacy and minimize immediate and long-term toxicity. The aim of this article is to present recent information that has immediate relevance for those who care for patients with Hodgkin's lymphoma Rev. Bras. Hematol. Hemoter. 2009;31(Supl. 2):3-6.

Key words: Hodgkin lymphoma; treatment; elderly; pregnancy.

\section{Referências Bibliográficas}

1.Diehl V, Sextro M, Franklin J, et al. Clinical presentation, course, and prognostic factors in lymphocyte-predominant Hodgkin's disease and lymphocyte-rich classical Hodgkin's disease: Report from the European Task Force on Lymphoma Project on Lymphocyte-Predominant Hodgkin's Disease. J Clin Oncol. 1999 17(3):776-83

2. Biasoli I, Stamatoullas A, Divine M, et al. A retrospective cohort of lymphocyte predominant Hodgkin lymphoma: long-term outcome and the risk of transformation into diffuse large-B cell lymphoma. Ann Oncol 2008; 19 (Supl 4), iv 112.

3. Brepoels L, Stroobants S. Is [18F]fluorodeoxyglucose positron emission tomography the ultimate tool for response and prognosis assessment? Hematol Oncol Clin North Am. 2007;21(5):855-69.

4. Gallamini A, Hutchings M, Rigacci L, et al. Early interim 2[18F]fluoro-2-deoxy-D-glucose positron emission tomography is prognostically superior to international prognostic score in advanced-stage Hodgkin's lymphoma: A report from a joint ItalianDanish study. J Clin Oncol. 2007;25(24):3746-52.

5. Gallamini A, Fiore F, Sorasio R, et al. Early chemotherapy intensification with BEACOPP in high-risk, ínterim-positive, advanced-stage Hodgkin lymphoma, improves the overall treatment outcome of ABVD: A GITIL multicenter clinical study. Haematologica 2009;94(s2):204.

6. Brepoels L, Stroobants S. PET scanning and prognosis in Hodgkin's lymphoma. Curr Op Oncol. 2008;20:509-16.

7. Eichenauer DA, Engert A. Recent advances in the treatment of early-stage Hodgkin's lymphoma. Education Program for the 14th congress of the European Hematology Association. 2009;3(1), 151-4.

8. Engert A, Pluetschow A, Eich HT, et al. Combined modality treatment of two or four cycles of ABVD followed by involved field radiotherapy in the treatemnt of patients with earlyl stage Hodgkin's lymphoma: update interim analysis of the randomised HD10 study of the German Hodgkin Study Group (GHSG). Blood. $2005 ; 106,2673 a$

9. Engert A, Borchmann P, Pluetschow A, et al. Dose-intensified combined modality treatment with 2 Cycles of BEACOPP escalated followed by 2 cycles of ABVD and involved field radiotherapy (IFRT) Is superior to 4 cycles of ABVD and IF-RT in patients with early unfavourable hodgkin lymphoma (HL): An Analysis of the German Hodgkin Study Group (GHSG) HD14 Trial. Haematologica 2009; 94 (s2), 226

10. Meyer RM, Gospodarowicz MK, Connors JM et al. Randomized comparison of ABVD chemotherapy with a strategy that includes radiation therapy in patients with limited-stage Hodgkin's lymphoma: National Cancer Institute of Canada Clinical Trials 
Group and the Eastern Cooperative Oncology Group. J Clin Oncol. 2005;23(21):4634-42.

11.Straus DJ, Portlock CS, Qin J, et al. Results of a prospective randomized clinical trial of doxorubicin, bleomycin, vinblastine, and dacarbazine (ABVD) followed by radiation therapy (RT) versus ABVD alone for stages I, II, and IIIA nonbulky Hodgkin's disease. Blood. 2004;104:3483-89.

12. Campbell BA, Voss N, Pickles T, et al. Involved-nodal radiation therapy as a component of combination therapy for limited-stage Hodgkin's Lymphoma: A question of field size. J Clin Oncol. 2008; 26:5170-74.

13. Martin WG, Ristow KM, Habermann TH, et al. Bleomycin pulmonary toxicity has a negative impact on the outcome of patients with Hodgkin's Lymphoma J Clin Oncol. 23:7614-7620.

14. Evens AM, Cilley J, Ortiz T, Gounder M, Hou N, Rademaker A, et al. G-CSF is not necessary to maintain over $99 \%$ dose-intensity with ABVD in the treatment of Hodgkin lymphoma: low toxicity and excellent outcomes in a 10-year analysis. British Journal of Haematology. 2007;137(6):545-52.

15. Proctor SJ, Rueffer JU, Angus B, et al. Hodgkin's disease in the elderly: current status and future directions. Ann Oncol. 2002;13 (suppl 1):133-7.

16. Bachanova V, Connors JM How Is Hodgkin Lymphoma in Pregnancy Best Treated? ASH Evidence-based Review, Educational Book, 2008.

O tema apresentado foi proposto pela Organização do $8^{\circ}$ Simpósio da Associação Ítalo-Brasileira de Hematologia, realizado durante o XIII Congresso de Transplante de Medula Óssea.

Publicado após concordância do editor.

Conflito de interesse: sem conflito de interesse

Recebido: 19/06/2009

Aceito: 08/07/2009 